\title{
Improved affine estimates for nonlinear viscoelastic composites
}

\author{
R. Brenner ${ }^{\mathrm{a}, *}$, R. Masson ${ }^{\mathrm{b}}$ \\ ${ }^{a}$ Laboratoire des Propriétés Mécaniques et Thermodynamiques des Matériaux, CNRS, Université Paris Nord, 93430 Villetaneuse, France \\ ${ }^{\mathrm{b}}$ Electricité de France, Division Recherche et Développement, Les Renardières, 77818 Moret sur Loing, France
}

Received 4 October 2004; accepted 10 June 2005

Available online 2 August 2005

\begin{abstract}
Motivated by recent results obtained in the context of variational approaches, new estimates are proposed for the homogenised behaviour of nonlinear viscoelastic materials. The key point relies in the introduction of field fluctuations in the context of hereditary behaviours. This is accomplished by incorporating the intraphase second moment of the mechanical fields of a reference linear viscoelastic material in an hereditary linearisation procedure. Analytical results for the evolution of field fluctuations within a linear viscoelastic composite are derived. Some illustrative results are presented for the creep response of a nonlinear viscoelastic matrix containing elastic inclusions with different morphologies. Compared with a classical approach, the proposed procedure leads to a softer overall response for both transient and steady-state regimes, and predicts a faster stress field redistribution.
\end{abstract}

() 2005 Elsevier SAS. All rights reserved.

Keywords: Nonlinear viscoelasticity; Composite; Homogenisation

\section{Introduction}

This article deals with the derivation of new estimates, in the context of the homogenisation theory, for the overall mechanical response of an heterogeneous nonlinear viscoelastic material. Significant advances have been recently made for such nonlinear composites which exhibit a coupling between a conservative and a dissipative constitutive mechanism. On one hand, Rougier et al. (1994) and Masson and Zaoui (1999) showed that the pioneering work of Laws and McLaughlin (1978) in the linear context, based on the use of the correspondence principle (Mandel, 1966) and the Laplace-Carson (LC) transform technique, could be extended to nonlinear behaviours. The subsequent work of Pouya and Zaoui (1999) has shown that this so-called "affine" formulation constitutes the first order approximation of a general linearisation procedure for which the local behaviour is linearised with respect to the whole stress (or strain) history up to the current time $t$. On the other hand, for behaviours deriving from a single potential (nonlinear elasticity, for instance), the importance of the field fluctuations for the derivation of the overall behaviour has been pointed out by numerous studies (see Ponte Castañeda and Suquet, 1998). Especially, it has been observed that a wide variety of estimates neglecting the intraphase field fluctuations leads to unrealistic estimates of the effective energy with respect to rigorous nonlinear bounds (Gilormini, 1995). Owing to these results, the question of an homogenisation scheme for nonlinear viscoelasticity that would account for field fluctuations is relevant. The present study is an attempt towards this aim.

\footnotetext{
* Corresponding author.

E-mail address: rb@galilee.univ-paris13.fr (R. Brenner).
} 
The paper is composed as follows. First, the computation of the field fluctuations for a linear viscoelastic composite is detailed. In a simple case allowing to derive exact analytical results, an approximate method for the inverse LC transform, namely the "direct inversion method" (DIM), is discussed. Then in the nonlinear framework, after a brief recall of the affine procedure, different possibilities for incorporating the available statistical information on the field fluctuations are presented. Particularly, an improved affine estimate is constructed by defining the linearised viscous compliance according to the new second-order method (Ponte Castañeda, 2002a) whereas the classical affine estimate uses the definition from the old secondorder approach (Ponte Castañeda, 1996). The obtained scheme is further simplified by using the DIM previously studied in the linear framework. The different nonlinear extensions are then compared for the case of an isotropic two-phase composite with particulate microstructure. Throughout this paper, we consider a viscoelastic microstructurally random composite material. This heterogeneous medium occupies a volume $\Omega$ and is made of $N$ constituents phases $(r)$, perfectly bonded, occupying volumes $\Omega_{r}$ (volumic fraction equals to $c_{r}$ ).

\section{Field fluctuations in linear viscoelastic composites}

Hereafter, the phases of the composite medium obey a non-ageing linear viscoelastic behaviour. This material is subjected to a known macroscopic mechanical loading for time $t \in\left[0 ;+\infty\left[\right.\right.$. At a point $\mathbf{x} \in \Omega_{r}$, the stress-strain behaviour obeys the following integral relation

$$
\boldsymbol{\varepsilon}(\mathbf{x}, t)=\int_{0}^{t} \mathbf{q} r(t-u): \mathrm{d} \boldsymbol{\sigma}(\mathbf{x}, u)
$$

with $\mathbf{q}_{r}(t)$ the tensorial creep function and $\int_{0}^{t} f(t-u): \mathrm{d} g(u)$ the Stieljes convolution product.

\subsection{Analytical computation of second moments}

Linear non-ageing viscoelastic problems can be reduced to linear elastic ones by the use of the LC transform defined as

$$
f^{*}(p)=p \int_{0}^{+\infty} f(t) \mathrm{e}^{-p t} \mathrm{~d} t,
$$

with $p$ the complex variable, $f$ the original time function and $f^{*}$ its LC transform.

Therefore, application of the LC transform to relation (1) gives a linear elastic relation between LC transforms of strain and stress histories, namely

$$
\boldsymbol{\varepsilon}^{*}(\mathbf{x}, p)=\mathbf{q}_{r}^{*}(p): \boldsymbol{\sigma}^{*}(\mathbf{x}, p)
$$

where $\mathbf{q}_{r}^{*}(p)$ denotes the (symbolic) linear elastic compliance of any phase $(r)$.

For a given real value of $p$, we now consider the heterogeneous composite whose microstructure is the same as the previous one but with phases obeying the linear elastic behaviour defined by (3). Classical homogenisation schemes for linear behaviours apply to this new composite. Its overall compliance is $\mathbf{Q}^{*}(p)$ while the overall stress applied to this composite is $\boldsymbol{\Sigma}^{*}(p)$.

Stress field second moments through any phase $(r)$ of this linear elastic composite are defined by

$$
\left\langle\boldsymbol{\sigma}^{*} \otimes \boldsymbol{\sigma}^{*}\right\rangle_{r, p}=\left\langle\boldsymbol{\sigma}^{*}(p) \otimes \boldsymbol{\sigma}^{*}(p)\right\rangle_{r}
$$

where the notation $\langle\cdot\rangle_{r}$ denotes the average on volume $\Omega_{r}$ of phase $(r)$. They can be obtained from any linear homogenisation scheme by computing the partial derivatives of the stress elastic energy of the composite with respect to the fourth-order tensor $\mathbf{q}_{r}^{*}(p)$ (Ponte Castañeda and Suquet, 1998)

$$
\left\langle\boldsymbol{\sigma}^{*} \otimes \boldsymbol{\sigma}^{*}\right\rangle_{r, p}=\frac{1}{c_{r}}\left[\boldsymbol{\Sigma}^{*}(p) \otimes \boldsymbol{\Sigma}^{*}(p):: \frac{\partial \mathbf{Q}^{*}(p)}{\partial \mathbf{q}_{r}^{*}(p)}\right] .
$$

Furthermore, according to the correspondence principle (Mandel, 1966), the second moment of the stress field throughout the original viscoelastic composite's phase is given by

$$
\langle\boldsymbol{\sigma} \otimes \boldsymbol{\sigma}\rangle_{r, t}=\langle\boldsymbol{\sigma}(t) \otimes \sigma(t)\rangle_{r}=\mathrm{LC}^{-1}\left\{\left\langle\boldsymbol{\sigma}^{*} \otimes \boldsymbol{\sigma}^{*}\right\rangle_{r, p}\right\}
$$

with $\mathrm{LC}^{-1}$ denoting the inverse operator to LC transform defined by: $\mathrm{LC}^{-1}\{\mathrm{LC}[f(t)]\}=f(t)$. 
Except for some limited situations (see Section 2.2), the explicit determination of the LC inverse operator is not straightforward and a numerical inversion based on finite Dirichlet series development is required (see the collocation method proposed by Schapery, 1962). In the following, we aim at applying an approximate analytic LC transform inversion method, the so-called "direct inversion method" (Schapery, 1962; Brenner et al., 2002) which provides a simple non-integral relation between the function and its transform. Indeed, its application to (6) yields

$$
\langle\boldsymbol{\sigma} \otimes \boldsymbol{\sigma}\rangle_{r, t} \approx\left\langle\boldsymbol{\sigma}^{*} \otimes \boldsymbol{\sigma}^{*}\right\rangle_{r, p=10^{w_{0} / t}}
$$

the choice of $w_{0}$ depending on phase constitutive behaviour as well as deformation path (Brenner et al., 2002). The value $w_{0}=0$ has been proved to be the best one for Maxwellian phase behaviours and creep responses. In the next section, we show that this choice is the most appropriate for the present study which is mainly focused on the creep response of nonlinear viscoelastic heterogeneous composites.

\subsection{Application}

We consider an isotropic material whose constituents obey an isotropic incompressible Maxwellian behaviour defined by

$$
\dot{\boldsymbol{\varepsilon}}(t)=\mathrm{m}_{r}^{\mathrm{e}} \dot{\mathbf{s}}(t)+\mathrm{m}_{r}^{\mathrm{v}} \mathbf{s}(t) .
$$

The scalar constants $\mathrm{m}_{r}^{\mathrm{e}}$ and $\mathrm{m}_{r}^{\mathrm{V}}$ are, respectively, the elastic and viscous shear compliances of phase $(r), \dot{\boldsymbol{\varepsilon}}$ is isochoric and $\mathbf{s}$ is the stress deviator. Thus, the local relaxation spectrum reduces to a single relaxation time $\tau_{r}=\mathrm{m}_{r}^{\mathrm{e}} / \mathrm{m}_{r}^{\mathrm{V}}$ and the scalar creep function of any phase $(r)$ reads: $\mathrm{q}_{r}(t)=\mathrm{m}_{r}^{\mathrm{e}}+t \mathrm{~m}_{r}^{\mathrm{V}}$. As previously, relation (8) can be transformed into

$$
\boldsymbol{\varepsilon}^{*}(p)=\mathrm{q}_{r}^{*}(p) \mathbf{s}^{*}(p)
$$

with $\mathrm{q}_{r}^{*}(p)=\mathrm{m}_{r}^{\mathrm{e}}+(1 / p) \mathrm{m}_{r}^{\mathrm{V}}$.

For the coming applications, we consider matrix-inclusion microstructures. By considering the connectedness of the matrix and restricting the study to low volume fractions of inclusions (up to 0.2), the overall behaviour and local mechanical fields are estimated through the classical Mori-Tanaka model (Mori and Tanaka, 1973) which identifies in the present case with the lower Hashin-Shtrikman bound. Note that the inclusions are assumed ellipsoidal and distributed within the material with the same ellipsoidal symmetry. In this specific context, the estimate of the time-dependent overall compliance as well as the first and second moments of the stress field within each phase can be derived analytically. The accuracy of the DIM can thus be checked rigorously at the global and local scales.

The exact and approximate analytic derivations lead to the following expressions for the overall viscoelastic compliance (sub-indice " 2 " refers to the matrix)

$$
\begin{aligned}
& \mathbf{Q}(t)^{\text {Exact }}=a\left[\mathrm{~m}_{2}^{\mathrm{ve}}(t)+(b-1)\left(\mathrm{m}_{2}^{\mathrm{v}} t+\left(\mathrm{m}_{2}^{\mathrm{e}}-\mathrm{m}_{2}^{\mathrm{v}} \tilde{\tau}\right)\left(1-\mathrm{e}^{-t / \tilde{\tau}}\right)\right)\right], \\
& \mathbf{Q}(t)^{\mathrm{DIM}}=a\left[\mathrm{~m}_{2}^{\mathrm{ve}}(t)+(b-1) \mathrm{m}_{2}^{\mathrm{ve}}(t) \frac{t}{t+\tilde{\tau}}\right] .
\end{aligned}
$$

For a creep loading path (i.e. constant overall stress, $\mathbf{S}(t)=\mathbf{S}_{0} \forall t \geqslant 0$ ), the average stress field in the matrix reads

$$
\begin{aligned}
& {\left[\langle\mathbf{s}(t)\rangle_{2}\right]_{\mathrm{eq}}^{\mathrm{Exact}}=d\left\{1+\left(\frac{\tilde{\tau}}{\hat{\tau}}-1\right)\left(1-\mathrm{e}^{-t / \tilde{\tau}}\right)\right\} \mathbf{S}_{0},} \\
& {\left[\langle\mathbf{s}(t)\rangle_{2}\right]_{\mathrm{eq}}^{\mathrm{DIM}}=d\left\{1+\left(\frac{\tilde{\tau}}{\hat{\tau}}-1\right) \frac{t}{t+\tilde{\tau}}\right\} \mathbf{S}_{0}}
\end{aligned}
$$

and the second moment of the stress field in the matrix reads

$$
\begin{aligned}
& \left\langle\left. s_{\text {eq }}^{2}(t)\right|_{2} ^{\text {Exact }}=\frac{1}{5-3 c_{2}}\left\{2+c\left[1+2\left(\frac{\tilde{\tau}}{\tau_{1}}-1\right)\left(1-\mathrm{e}^{-t / \tilde{\tau}}\right)+\left(\frac{\tilde{\tau}}{\tau_{1}}-1\right)^{2}\left(1-\left(\frac{t+\tilde{\tau}}{\tilde{\tau}}\right) \mathrm{e}^{-t / \tilde{\tau}}\right)\right]\right\} \mathbf{S}_{0}^{2},\right. \\
& \left\langle\left. s_{\mathrm{eq}}^{2}(t)\right|_{2} ^{\mathrm{DIM}}=\frac{1}{5-3 c_{2}}\left\{2+c\left[1+2\left(\frac{\tilde{\tau}}{\tau_{1}}-1\right) \frac{t}{t+\tilde{\tau}}+\left(\frac{\tilde{\tau}}{\tau_{1}}-1\right)^{2} \frac{t^{2}}{(t+\tilde{\tau})^{2}}\right]\right\} \mathbf{S}_{0}^{2} .\right.
\end{aligned}
$$




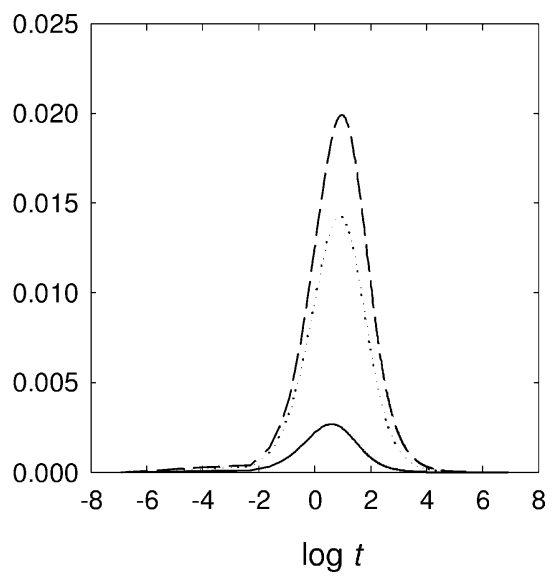

Fig. 1. Relative error between analytic and "direct" inverse transforms of the overall creep function as a function of $\log t$ (continuous: $\tau_{1} / \tau_{2}=2$, dotted: $\tau_{1} / \tau_{2}=10$, dashed: $\left.\tau_{1} / \tau_{2}=100\right)$.

Note that the following constants have been defined

$$
\begin{cases}a=\frac{5 \mathrm{~m}_{1}^{\mathrm{e}}+2 c_{2}\left(\mathrm{~m}_{2}^{\mathrm{e}}-\mathrm{m}_{1}^{\mathrm{e}}\right)}{3 c_{2} \mathrm{~m}_{1}^{\mathrm{e}}+\left(5-3 c_{2}\right) \mathrm{m}_{2}^{\mathrm{e}},} & b=\tilde{\tau} \frac{5 \mathrm{~m}_{1}^{\mathrm{v}}+2 c_{2}\left(\mathrm{~m}_{2}^{\mathrm{v}}-\mathrm{m}_{1}^{\mathrm{v}}\right)}{5 \mathrm{~m}_{1}^{\mathrm{e}}+2 c_{2}\left(\mathrm{~m}_{2}^{\mathrm{e}}-\mathrm{m}_{1}^{\mathrm{e}}\right)}, \\ c=\frac{75\left(1-c_{2}\right) \mathrm{m}_{1}^{\mathrm{e} 2}}{\left(3 c_{2} \mathrm{~m}_{1}^{\mathrm{e}}+\left(5-3 c_{2}\right) \mathrm{m}_{2}^{\mathrm{e}}\right)^{2}}, & d=\frac{3 \mathrm{~m}_{1}^{\mathrm{e}}+2 \mathrm{~m}_{2}^{\mathrm{e}}}{3 c_{2} \mathrm{~m}_{1}^{\mathrm{e}}+\left(5-3 c_{2}\right) \mathrm{m}_{2}^{\mathrm{e}}}, \\ \frac{1}{\tilde{\tau}}=\frac{3 c_{2} \mathrm{~m}_{1}^{\mathrm{v}}+\left(5-3 c_{2}\right) \mathrm{m}_{2}^{\mathrm{v}}}{3 c_{2} \mathrm{~m}_{1}^{\mathrm{e}}+\left(5-3 c_{2}\right) \mathrm{m}_{2}^{\mathrm{e}},} & \frac{1}{\hat{\tau}}=\frac{3 \mathrm{~m}_{1}^{\mathrm{v}}+2 \mathrm{~m}_{2}^{\mathrm{v}}}{3 \mathrm{~m}_{1}^{\mathrm{e}}+2 \mathrm{~m}_{2}^{\mathrm{e}}} .\end{cases}
$$

It is worth emphasising that elastic $(t \rightarrow 0)$ as well as purely viscous $(t \rightarrow+\infty)$ responses derived using the DIM are exact.

Comparisons between the exact overall viscoelastic compliance (i.e. creep function) and the one obtained with the DIM are reported on Fig. 1 for different ratios of the two phases relaxation times $\left(2 \leqslant \tau_{1} / \tau_{2} \leqslant 100, \mathrm{~m}_{1}^{\mathrm{e}} / \mathrm{m}_{2}^{\mathrm{e}}=1\right)$. It is worth mentioning that the DIM leads to the exact LC inversion result when the two phases have identical relaxation times whatever the value of the elastic contrast $\mathrm{m}_{1}^{\mathrm{e}} / \mathrm{m}_{2}^{\mathrm{e}}$ (Brenner et al., 2002). Consequently, the accuracy of the DIM is especially dependent on the contrast of the relaxation times of the two phases. Results derived with the direct method are very close to exact ones in transient regimes even for large contrasts $\left(\tau_{1} / \tau_{2}=100\right)$. This good agreement for creep loading is consistent with former simulations (Brenner et al., 2002) derived with other homogenisation schemes (Voigt as well as self-consistent schemes) associated to different microstructures ("parallel" as well as polycrystalline microstructures). Similar comparisons concerning variations with time of both first and second moments of the stress field throughout the matrix are reported on Fig. 2. Deviations between exact and direct inversions remain still acceptable (less than $8 \%$ ) for the various contrasts studied.

\subsection{Conclusions}

The correspondence principle reduces the computation of field fluctuations of stress (or strain) in non-ageing linear viscoelastic composites to the LC inversion of corresponding field fluctuations throughout a composite whose microstructure is the same as the previous one but with phases obeying a linear elastic behaviour. LC transform of field fluctuations can then be obtained with any homogenisation scheme by computing the partial derivatives of the stress (or strain) elastic energy with respect to the local compliance (or stiffness).

To compute the inverse LC transform, the DIM provides an approximate but powerful way. This method has been applied to simple isotropic matrix-inclusion composite with linear Maxwellian local behaviour. For creep loadings, the creep function as well as the evolutions with time of first and second moments of the stress field in the matrix have been computed analytically (Mori-Tanaka estimates). Comparisons between these exact results and the ones derived with the DIM display low deviations even for high contrasts (two phases relaxation time ratios up to 100). Consequently, it can be advantageously used for nonlinear problems which reduce, at each linearisation time, to a new linear problem. As shown in the next section, the application of the DIM gives rise to a so-called "quasi-elastic" formulation of the affine model for nonlinear viscoelasticity. 


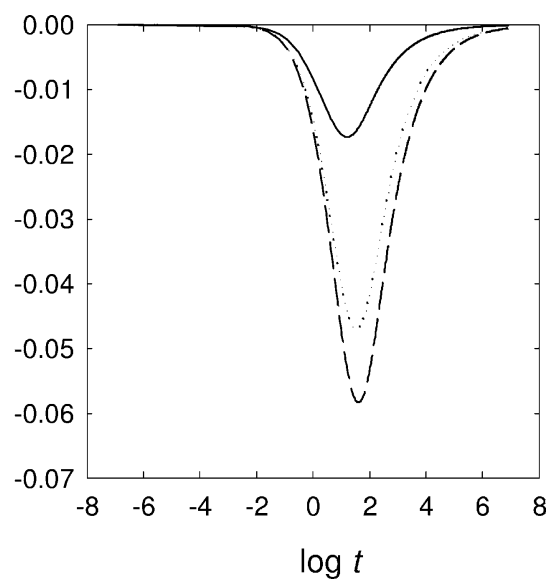

(a)

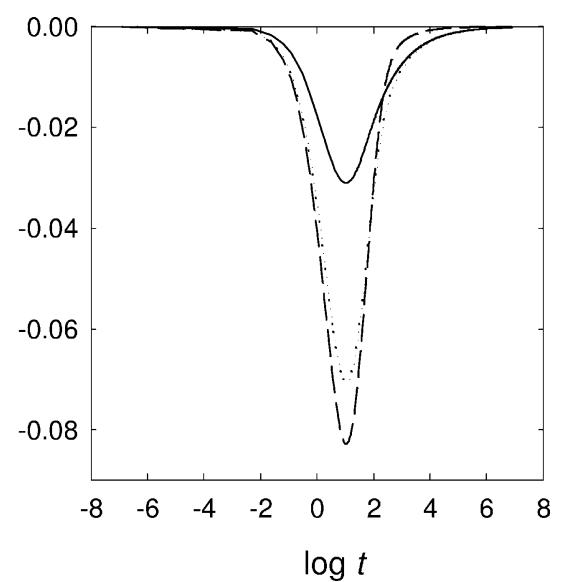

(b)

Fig. 2. Relative error between analytic and "direct" inverse transforms of (a) the average stress field and (b) the second moment of the equivalent stress in the matrix as a function of $\log t$ (continuous: $\tau_{1} / \tau_{2}=2$, dotted: $\tau_{1} / \tau_{2}=10$, dashed: $\tau_{1} / \tau_{2}=100$ ).

\section{Homogenisation procedure for nonlinear viscoelastic composites}

A microstructurally random composite material, with linear compressible elasticity and nonlinear incompressible viscosity, is now considered. The constitutive behaviour of the individual constituents is governed by two stress-energy functions $u^{e}(\sigma)$, for the elasticity, and $u^{v}(\mathbf{s})$, for the viscosity, which present respectively a quadratic and a more than quadratic growth. At each point $\mathbf{x}$ inside $\Omega$, the infinitesimal local strain rate $\dot{\varepsilon}$ reads

$$
\dot{\boldsymbol{\varepsilon}}(t)=\frac{\mathrm{d}}{\mathrm{d} t}\left(\frac{\partial u^{\mathrm{e}}}{\partial \boldsymbol{\sigma}}(\boldsymbol{\sigma}(t))\right)+\frac{\partial u^{\mathrm{v}}}{\partial \mathbf{s}}(\mathbf{s}(t)) .
$$

With use of a functional representation, this local constitutive equation can be expressed as

$$
\boldsymbol{\varepsilon}(t)=\mathcal{G}\left\{\begin{array}{c}
t \\
\sigma(\theta) \\
0
\end{array}\right\}
$$

where $\mathcal{G}$ is a nonlinear functional which correlates the strain $\boldsymbol{\varepsilon}(t)$ at a given time $t$ with the whole stress history $\boldsymbol{\sigma}(\theta)$ with $0 \leqslant \theta \leqslant t$.

\subsection{Hereditary affine formulation}

The specificity of the required linearisation procedure for nonlinear viscoelastic behaviours lies in the differential nature of the constitutive relation (14) which reads alternatively

$$
\dot{\boldsymbol{\varepsilon}}(\mathbf{x}, t)=\mathbf{m}^{\mathrm{e}}(\mathbf{x}): \dot{\boldsymbol{\sigma}}(\mathbf{x}, t)+\mathbf{g}(\mathbf{x}, \mathbf{s}(\mathbf{x}, t)), \quad \forall \mathbf{x} \in \Omega,
$$

with $\mathbf{m}^{\mathrm{e}}$ the elastic compliance and $\mathbf{g}$ the nonlinear viscous strain rate deriving from the potential $u^{\mathrm{v}}$. At each time $t$, the dependence of the strain rate on the whole stress history from $t=0$ prevents from a direct definition of instantaneous moduli or compliances as it is the case for nonlinear purely elastic or viscous behaviours. To treat this difficulty, Rougier et al. (1994) initially proposed a linearisation procedure along the loading path which reads

$$
\dot{\boldsymbol{\varepsilon}}(t)=\mathbf{m}^{\mathrm{e}}: \dot{\boldsymbol{\sigma}}(t)+\mathbf{m}^{\mathrm{v}}(\zeta): \mathbf{s}(t)+\dot{\boldsymbol{\varepsilon}}^{0}(t, \zeta)
$$

with

$$
\begin{cases}\dot{\boldsymbol{\varepsilon}}^{0}(t, \zeta)=\mathbf{g}(\mathbf{s}(t))-\mathbf{m}^{\mathrm{v}}(\zeta): \mathbf{s}(t) & \forall t \leqslant \zeta \\ \dot{\boldsymbol{\varepsilon}}^{0}(t, \zeta)=\mathbf{g}(\mathbf{s}(\zeta))-\mathbf{m}^{\mathrm{v}}(\zeta): \mathbf{s}(\zeta) & \forall t \geqslant \zeta\end{cases}
$$

It can be remarked that the stress-free strain rate $\dot{\boldsymbol{\varepsilon}}^{0}(t, \zeta)$ does not depend on the mechanical loading after $t=\zeta$. According to (17) and (18), this procedure thus leads to the definition of a linear thermoviscoelastic heterogeneous medium. At this point, it is worth noting that there is no requirement for the linearised viscous compliance $\mathbf{m}^{\mathrm{v}}(\zeta)$ to correspond to the tangent compliance

$$
\mathbf{m}^{\operatorname{tgt}}(\zeta)=\frac{\partial \mathbf{g}}{\partial \mathbf{s}}(\mathbf{s}(\zeta))
$$


Alternatively, relation (17) can be put in the following integral form

$$
\left\{\begin{array}{l}
\boldsymbol{\varepsilon}(t)=\int_{0}^{t} \mathbf{q}_{\zeta}(t-u): \mathrm{d} \boldsymbol{\sigma}(u)+\boldsymbol{\varepsilon}^{0}(t, \zeta), \\
\mathbf{q}_{\zeta}(t)=\mathbf{m}^{\mathrm{e}}+\mathbf{m}^{\mathrm{v}}(\zeta) t, \\
\boldsymbol{\varepsilon}^{0}(t, \zeta)=\int_{0}^{t} \dot{\boldsymbol{\varepsilon}}^{0}(\theta, \zeta) \mathrm{d} \theta
\end{array}\right.
$$

where it is emphasised that the stress-free strain $\varepsilon^{0}$ is a function of the whole stress history thus accounting for the viscoelastic coupling phenomena within the material.

\subsection{Uniform behaviour per phase}

We restrict ourselves to the derivation of estimates for the overall behaviour using classical mean-field approaches. Using a statistical description of the microstructure, through the first-order (i.e. volume fractions) and second-order (i.e. covariances) spatial correlation functions, it consists in the determination of the phase stress and strain averages, $\langle\boldsymbol{\sigma}\rangle_{r}$ and $\langle\boldsymbol{\varepsilon}\rangle_{r}$. This can be achieved by using Eshelby's solution (Eshelby, 1957) of the inclusion problem. Nevertheless, in the nonlinear context, this step itself is not straightforward. Indeed, the dependence of the local behaviour on the mechanical fields, which vary continuously in the material, leads to nonuniform intraphase responses and implies

$$
\langle\boldsymbol{\varepsilon}(t)\rangle_{r}=\left\langle\mathcal{G}\left\{\begin{array}{c}
t \\
\boldsymbol{\sigma}(\theta) \\
0
\end{array}\right\}\right\rangle_{r} \neq \mathcal{G}\left\{\begin{array}{c}
t \\
\langle\boldsymbol{\sigma}(\theta)\rangle_{r} \\
0
\end{array}\right\} .
$$

In any phase, the stress and strain average histories are not linked by the constitutive equation of the phase. By contrast to the case of a linear behaviour, the resolution of the homogenisation problem thus requires complementary relations to define an uniform behaviour per phase. For this crucial step, use has to be made of the statistical information, delivered by linear homogenisation schemes, on the mechanical fields distribution. The available quantities are the intraphase averages given by the linear concentration equations, and the intraphase second moments obtained by derivation of the overall energy with respect to the uniform local compliances (or moduli). Initially derived in the case of isotropic elasticity (Bobeth and Diener, 1986), the field intraphase second moments have subsequently been given in more general cases (Kreher, 1990; Buryachenko, 1993, 2001; Ponte Castañeda and Suquet, 1998). These intraphase fluctuations result from the interphase interactions inside the material. It is stressed that the adopted definition for the linearised behaviour per phase will give rise to distinct estimations for the overall behaviour as well as for the fields distribution. The present work is focused on different possibilities in the framework of the hereditary affine procedure presented above.

\subsubsection{Original affine estimates}

The initial proposal made by Rougier et al. (1994) and Masson and Zaoui (1999) consists in considering a tangent linearisation defined with respect to the average fields over each phase. The linearised viscous compliance reads

$$
\mathbf{m}_{r}^{\mathrm{v}}=\frac{\partial \mathbf{g}}{\partial \mathbf{s}}\left(\langle\mathbf{s}\rangle_{r}\right) \text {. }
$$

This choice, consistent with the pioneering work of Hill (1965) for elastoplasticity, implies that the stress and strain average histories are linked by the constitutive behaviour. Clearly, this condition can only be fulfilled if the mechanical fields are homogeneous within each phase in the nonlinear material (e.g. laminate microstructures). In the general case, since intraphase heterogeneity is not accounted for in the definition of the linearised behaviour, it might be expected that this formulation presents some deficiencies especially for situations where the field fluctuations inside the material become important (high contrast and/or high nonlinearity) (Masson et al., 2000). We will refer to this approach as the "classical" affine (CA).

\subsubsection{Improved affine estimates}

In the context of variational approaches, Ponte Castañeda (2002a) recently proposed a theory which incorporates the field fluctuations for the derivation of the effective behaviour. This formulation still makes use of an affine linearisation. Nevertheless, instead of using a tangent compliance, it defines a "generalised" affine (GA) compliance which depends on both first and second moments of the intraphase fields. For purely nonlinear viscous behaviours, this procedure improves on previous proposals (Ponte Castañeda, 2002b; Idiart and Ponte Castañeda, 2003). In contradistinction with classical mean-field approaches, it has 
to be mentioned that this variational procedure does not use the linearised constitutive behaviour to link the average fields per phase. However, by dismissing this condition, Ponte Castañeda (2002a) proposed an extension of this approach to the classical mean-field context. Whereas it can be proved that the resulting estimate is less accurate than the corresponding stationary ones, it presents the specific advantage of being incorporable into the present viscoelastic procedure. It is worth noting that although this GA estimate does account for field fluctuations, through the linearised compliance, it still assumes that the stress and strain average histories are linked by the constitutive behaviour as the CA estimate does. According to this theory, the linearised viscous compliance is obtained from the following condition

$$
\frac{\partial u^{\mathrm{v}}}{\partial \mathbf{s}}\left(\hat{\mathbf{s}}_{r}\right)-\frac{\partial u^{\mathrm{v}}}{\partial \mathbf{s}}\left(\langle\mathbf{s}\rangle_{r}\right)=\mathbf{m}_{r}^{\mathrm{v}}:\left(\hat{\mathbf{s}}_{r}-\langle\mathbf{s}\rangle_{r}\right)
$$

with $\hat{\mathbf{s}}_{r}$ a reference deviatoric stress state. Hereafter, restriction is made to tensors $\mathbf{m}_{r}^{\mathrm{v}}$ whose principal axes are aligned with $\langle\mathbf{s}\rangle_{r}$, i.e.

$$
\mathbf{m}_{r}^{\mathrm{v}}=\frac{1}{2 \lambda_{0}^{r}} \mathbf{E}^{r}+\frac{1}{2 \mu_{0}^{r}} \mathbf{F}^{r}
$$

where use has been made of the projection operators $\mathbf{E}^{r}$ and $\mathbf{F}^{r}$ introduced in Ponte Castañeda (1996):

$$
\mathbf{E}^{r}=\frac{\langle\mathbf{s}\rangle_{r} \otimes\langle\mathbf{s}\rangle_{r}}{\langle\mathbf{s}\rangle_{r}:\langle\mathbf{s}\rangle_{r}} \quad \text { and } \quad \mathbf{F}^{r}=\mathbf{K}-\mathbf{E}^{r},
$$

with $\mathbf{K}$ the deviatoric isotropic projection tensor. The reference fields $\langle\mathbf{s}\rangle_{r}$ and $\hat{\mathbf{s}}_{r}$ are respectively given by the average stress localisation relation and the condition

$$
\left(\hat{\mathbf{s}}_{r}-\langle\mathbf{s}\rangle_{r}\right) \otimes\left(\hat{\mathbf{s}}_{r}-\langle\mathbf{s}\rangle_{r}\right)=\mathbf{C}_{\mathbf{s}}^{r}
$$

with $\mathbf{C}_{\mathbf{S}}^{r}=\langle\mathbf{s} \otimes \mathbf{s}\rangle_{r}-\langle\mathbf{s}\rangle_{r} \otimes\langle\mathbf{s}\rangle_{r}$ the covariance tensor of the deviatoric stresses in phase (r). Because of the choice (24), relation (26) reduces to

$$
\hat{s}_{\|}^{r}=\bar{\sigma}_{\mathrm{eq}}^{r}+\sqrt{\frac{3}{2} \mathbf{E}^{r}:: \mathbf{C}_{\mathbf{s}}^{r}} \text { and } \hat{s}_{\perp}^{r}=\sqrt{\frac{3}{2} \mathbf{F}^{r}:: \mathbf{C}_{\mathbf{s}}^{r}}
$$

with $\hat{s}_{\|}^{r}=\sqrt{\frac{3}{2} \hat{\mathbf{s}}_{r}: \mathbf{E}^{r}: \hat{\mathbf{s}}_{r}}, \hat{s}_{\perp}^{r}=\sqrt{\frac{3}{2} \hat{\mathbf{s}}_{r}: \mathbf{F}^{r}: \hat{\mathbf{s}}_{r}}$ and $\bar{\sigma}_{\mathrm{eq}}^{r}=\left[\langle\mathbf{s}\rangle_{r}\right]_{\text {eq }}$, i.e. the Von Mises equivalent of the average stress $\sqrt{\frac{3}{2}\langle\mathbf{s}\rangle_{r}:\langle\mathbf{s}\rangle_{r}}$. The stress second moment tensors $\langle\mathbf{s} \otimes \mathbf{s}\rangle_{r}$, required to compute $\mathbf{C}_{\mathbf{s}}^{r}$, are obtained by taking the partial derivative of the effective energy of the heterogeneous linearised medium with respect to the compliance of each phase $(r)$.

The present paper is mainly concerned with the two presented linearisation procedures. Nevertheless, to illustrate the variety of approaches that can be constructed, let us mention an alternative procedure proposed by Brenner et al. (2001) and aiming too at incorporating the field fluctuations for the derivation of the effective behaviour. This "modified" affine (MA) procedure defines the linearised compliance as the tangent compliance in the vicinity of the second moment of the intraphase field. On the other hand, the phase average strain rate $\langle\dot{\boldsymbol{\varepsilon}}\rangle_{r}$ is estimated by following the work of Suquet (1995). Consequently, this empirical formulation fulfils, by construction, the inequality relation in (21).

\section{3. "Quasi-elastic" approximation of the affine procedure}

By means of the adopted linearisation procedure, the treatment proposed by Laws and McLaughlin (1978) can be applied in the present nonlinear context at each linearisation time. It may be summarised in three steps:

- the thermoviscoelastic problem is converted into a symbolic thermoelastic problem using the Laplace transform technique,

- the linear homogenisation problem is solved,

- the solution is obtained in the time space by inverse transform of the result.

To perform the last step of this procedure, one can take advantage of the DIM introduced in Section 2. It is recalled that this approximation relies on a mathematical property of the Laplace transform which allows to identify (with a change of variables) a function with its transform. As a consequence, the homogenisation can be performed directly in the time space. This approach is relevant for different types of loading paths and especially for creep conditions. It is worth to note that this simplified treatment does retain the main features of the viscoelastic behaviour. In particular, the hereditary nature is kept and the overall response is not assumed to be of the Maxwell-type. As shown in Section 2 in the linear context, it delivers reasonable estimates at both local and global scales. The "quasi-elastic" approach, adopted in the sequel, can be formulated as follows. Considering, e.g., 
a macroscopic stress loading path $\Sigma(t)$ on the time interval $[0, \zeta]$ and assuming that the problem was solved until $t=\zeta-\delta t$, the linearised behaviour reads, for a given linearisation time $\zeta$, at any time $t \leqslant \zeta$

$$
\left\{\begin{array}{l}
\boldsymbol{\varepsilon}_{r}(t)=\mathbf{q}_{\zeta}(t): \boldsymbol{\sigma}_{r}(t)+\boldsymbol{\varepsilon}_{r}^{0}(t, \zeta), \\
\mathbf{q}_{\zeta}^{r}(t)=\mathbf{m}_{r}^{\mathrm{e}}+\mathbf{m}_{r}^{\mathrm{V}}(\zeta) t, \\
\boldsymbol{\varepsilon}_{r}^{0}(t, \zeta)=\int_{0}^{t} \dot{\boldsymbol{\varepsilon}}_{r}^{0}(\theta, \zeta) \mathrm{d} \theta, \\
\dot{\boldsymbol{\varepsilon}}_{r}^{0}(\theta, \zeta)=\mathbf{g}\left(\mathbf{s}_{r}(\theta)\right)-\mathbf{m}_{r}^{\mathrm{V}}(\zeta): \mathbf{s}_{r}(\theta) .
\end{array}\right.
$$

According to the approximate procedure, it is only required to solve the homogenisation problem associated with (28) for $t=\zeta$. It reads

$$
\left\{\begin{array}{l}
\mathbf{Q}_{\zeta}(\zeta)=\left\langle\mathbf{q}_{\zeta}^{r}(\zeta): \mathbf{B}_{r}(\zeta)\right\rangle \\
\mathbf{E}^{0}(\zeta, \zeta)=\left\langle{ }^{t} \mathbf{B}_{r}(\zeta): \boldsymbol{\varepsilon}_{r}^{0}(\zeta, \zeta)\right\rangle
\end{array}\right.
$$

where $\mathbf{B}_{r}$ is the average stress concentration tensor. The interaction equation can be expressed as

$$
\left\{\begin{array}{l}
\boldsymbol{\varepsilon}_{r}(\zeta)-\mathbf{E}^{\prime}(\zeta)=-\widetilde{\mathbf{Q}}_{\zeta}(\zeta):\left(\sigma_{r}(\zeta)-\boldsymbol{\Sigma}^{\prime}(\zeta)\right) \\
\widetilde{\mathbf{Q}}_{\zeta}(\zeta)=\left(\left(\mathbf{S}^{E}\right)^{-1}-\mathbf{I}\right)^{-1}: \mathbf{Q}_{\zeta}^{*}(\zeta)
\end{array}\right.
$$

with $\widetilde{\mathbf{Q}}_{\zeta}$ the interaction tensor, $\mathbf{S}^{E}$ the Eshelby tensor, $\mathbf{Q}_{\zeta}^{*}$ the compliance of the reference medium which depends on the chosen homogenisation scheme. $\mathbf{E}^{\prime}$ and $\boldsymbol{\Sigma}^{\prime}$ are the homogeneous strain and stress fields in the infinite medium for the corresponding inclusion problem. They are linked to the macroscopic fields $\mathbf{E}$ and $\boldsymbol{\Sigma}$ by

$$
\begin{aligned}
& \mathbf{E}^{\prime}=\left(\tilde{\mathbf{L}}_{\zeta}+\mathbf{L}_{\zeta}^{*}\right)^{-1}:\left\langle\left(\tilde{\mathbf{L}}_{\zeta}+\mathbf{l}_{\zeta}^{r}\right)^{-1}\right\rangle^{-1}: \mathbf{E}, \\
& \boldsymbol{\Sigma}^{\prime}=\left(\widetilde{\mathbf{Q}}_{\zeta}+\mathbf{Q}_{\zeta}^{*}\right)^{-1}:\left\langle\left(\widetilde{\mathbf{Q}}_{\zeta}+\mathbf{q}_{\zeta}^{r}\right)^{-1}\right\rangle^{-1}: \mathbf{\Sigma}
\end{aligned}
$$

with $\tilde{\mathbf{L}}_{\zeta}=\widetilde{\mathbf{Q}}_{\zeta}^{-1}, \mathbf{L}_{\zeta}^{*}=\left(\mathbf{Q}_{\zeta}^{*}\right)^{-1}$ and $\mathbf{l}_{\zeta}^{r}=\left(\mathbf{q}_{\zeta}^{r}\right)^{-1}$, the linearisation time $\zeta$ being omitted for brevity. The homogenisation procedure defined by $(28),(29)$ and $(30)$, together with an adequate description of the microstructure, thus requires at each linearisation time $\zeta$ the resolution of a set of nonlinear equations which reads $F\left(\sigma_{r}(\zeta)\right)=0$.

\section{Illustrative applications: creep of a two-phase isotropic composite}

The different affine schemes introduced in Section 3 are now applied to the case of a two-phase composite presenting an isotropic constitutive relation. Within each phase $(r)$ of the composite, the local elastic and viscous stress potentials are characterised by

$$
\left\{\begin{array}{l}
u^{\mathrm{e}}(\mathbf{x})=\frac{\sigma_{\mathrm{eq}}^{2}(\mathbf{x})}{6 \mu_{r}^{\mathrm{e}}}+\frac{\sigma_{\mathrm{m}}^{2}(\mathbf{x})}{2 k_{r}^{\mathrm{e}}}, \\
u^{\mathrm{v}}(\mathbf{x})=\frac{\sigma_{0}^{r} \cdot \dot{e}_{0}}{n+1}\left(\frac{\sigma_{\mathrm{eq}}(\mathbf{x})}{\sigma_{0}^{r}}\right)^{n+1}=\psi\left(\sigma_{\mathrm{eq}}(\mathbf{x})\right)
\end{array}\right.
$$

with position vector $\mathbf{x} \in \Omega_{r} . \mu_{r}^{e}$ is the elastic shear modulus, $k_{r}^{e}$ the elastic bulk modulus and $\sigma_{0}^{r}$ the reference viscous stress, uniform per phase. $\dot{e}_{0}$ is a reference strain rate and $n$ the stress sensitivity coefficient, both homogeneous within the material. $\sigma_{\mathrm{m}}$ represents the hydrostatic stress defined by $\frac{1}{3} \operatorname{tr}(\sigma)$. For a given linearisation time $\zeta$, at any time $t \leqslant \zeta$, the linearised viscoelastic compliance obeys the following generic expression

$$
\left\{\begin{array}{l}
\mathbf{q}_{\zeta}^{r}(t)=\mathbf{m}_{r}^{\mathrm{e}}+\mathbf{m}_{r}^{\mathrm{v}}(\zeta) t \\
\mathbf{m}_{r}^{\mathrm{v}}(\zeta)=\alpha(\zeta) \mathbf{E}^{r}+\beta(\zeta) \mathbf{F}^{r}
\end{array}\right.
$$

The classical affine procedure leads to the following expressions for the scalars $\alpha$ and $\beta$

$$
\alpha(\zeta)=n \beta(\zeta), \quad \beta(\zeta)=\frac{3 \dot{e}_{0}}{2 \sigma_{0}^{r}}\left(\frac{\bar{\sigma}_{\mathrm{eq}}^{r}(\zeta)}{\sigma_{0}^{r}}\right)^{(n-1)}
$$




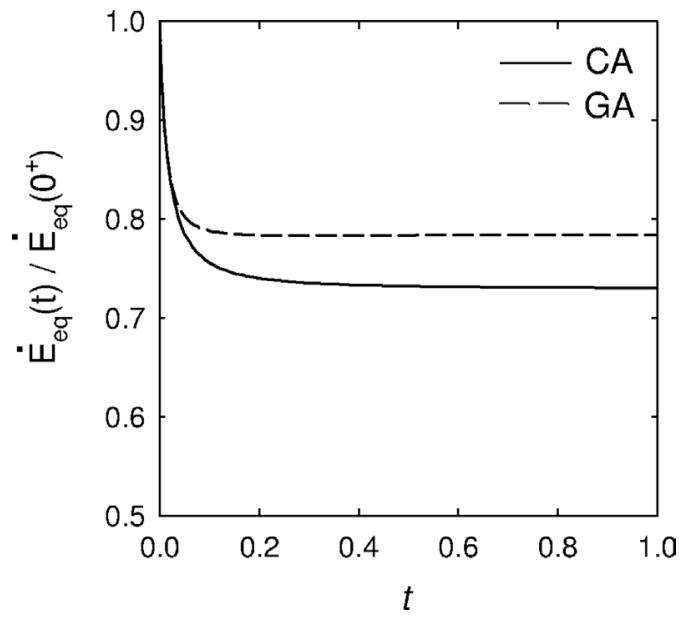

(a)

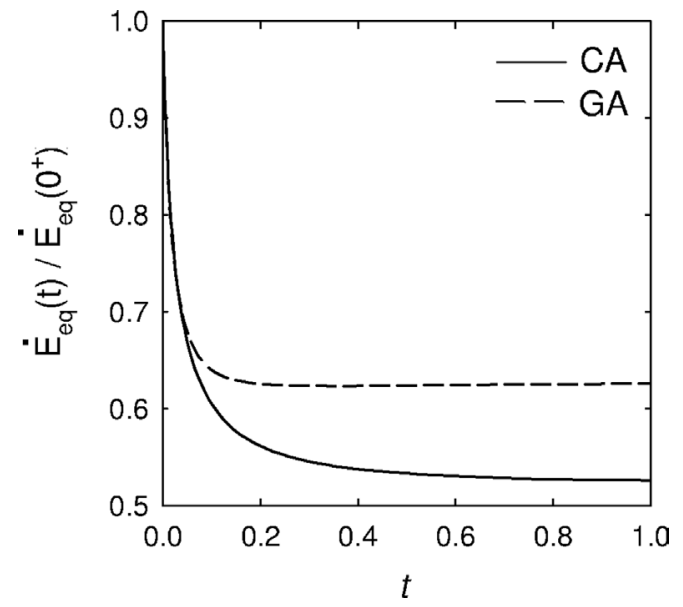

(b)

Fig. 3. Normalised overall equivalent strain rate estimated by the classical and generalised affine procedures for (a) aligned-fiber and (b) spherical inclusions. The dotted line corresponds to a decoupled Maxwell-type approach using the generalised affine procedure. The volumic fraction of inclusions is $c_{i}=0.15$ and the stress sensitivity exponent of the matrix is $n=5$. Case of a longitudinal simple shear with $\Sigma_{13} / \sigma_{0}^{(2)}=1$.

whereas the expressions obtained with the generalised affine procedure read

$$
\alpha(\zeta)=\frac{3}{2} \frac{\psi^{\prime}\left(\hat{\sigma}_{\mathrm{eq}}^{r}\right)\left(\hat{\sigma}_{\|}^{r} / \hat{\sigma}_{\mathrm{eq}}^{r}\right)-\psi^{\prime}\left(\bar{\sigma}_{\mathrm{eq}}^{r}\right)}{\left(\hat{\sigma}_{\|}^{r}-\bar{\sigma}_{\mathrm{eq}}^{r}\right)}, \quad \beta(\zeta)=\frac{3}{2} \frac{\psi^{\prime}\left(\hat{\sigma}_{\mathrm{eq}}^{r}\right)}{\hat{\sigma}_{\mathrm{eq}}^{r}}
$$

where the dependence on $\zeta$ of the tensors $\hat{\sigma}^{r}$ and $\bar{\sigma}^{r}$ has been omitted for brevity. Hereafter, the focus is on the special case of a viscoelastic matrix (phase 2) containing elastic inclusions (phase 1). Consequently, we have $\sigma_{0}^{(1)} \rightarrow \infty$ and thus $\mathbf{q}_{\zeta}^{(1)}(t)=\mathbf{m}_{(1)}^{\mathrm{e}}$. In the context of Mori-Tanaka estimates, the effect of the inclusions' morphology together with the loading path on the composite's overall response and its link with the local fields heterogeneity is now discussed for creep conditions.

\subsection{Creep under longitudinal shear}

In this case, two types of inclusions are considered: aligned (continuous) fibers (along $x_{3}$ axis) and spheres. We are interested in the response of the composite material subjected to (longitudinal) simple shear in $x_{3}$ direction. Note that the elastic properties are assumed uniform in the material so that we focus on the role of the viscous heterogeneity on the transient and steady-state (i.e. viscous) regimes. It is worth noting that, rigorously, this regime corresponds to the stabilisation of the stress field within the material. In our analysis, this condition is restricted to the first and second moments of the stress field within each constituent phase. Fig. 3 presents comparisons between CA and GA estimates for the evolution of the effective strain rate during creep. It is observed that the GA procedure predicts a weaker decrease of the strain rate during the test thus leading to a "softer" estimate of the steady-state regime in agreement with the results reported in Ponte Castañeda (2002b). In the transient regime, where the viscoelastic coupling takes place, it can be seen that the GA approach predicts a perceptibly shorter overall primary creep. In other words, to account for the heterogeneous character of the intraphase stress field leads to a faster stress field redistribution within the material. Note that a Maxwell-type approach neglecting the viscoelastic coupling would lead to a constant overall strain rate since the overall stress is constant $\forall t>0^{+}$. These observations are valid for parts (a) and (b) whereas the following differences deserve to be mentioned:

- spherical inclusions are responsible for a higher decrease of the effective strain rate independently of the approach,

- the relative discrepancy between CA and GA estimates is less pronounced for the microstructure with aligned fibers.

The interphase distribution of the average stress fields is reported in Fig. 4. The evolution of the average stress within each phase during the transient regime is characterised by a high increase in the inclusions which are purely elastic and a relatively moderate decrease in the matrix which undergoes a viscoelastic deformation. For both microstructures, the GA estimates lead to a lower stress interphase heterogeneity throughout the creep deformation. In agreement with the effect of the microstructure at the macroscopic scale, it can be seen that the spherical inclusions induce a higher stress interphase heterogeneity for a given 


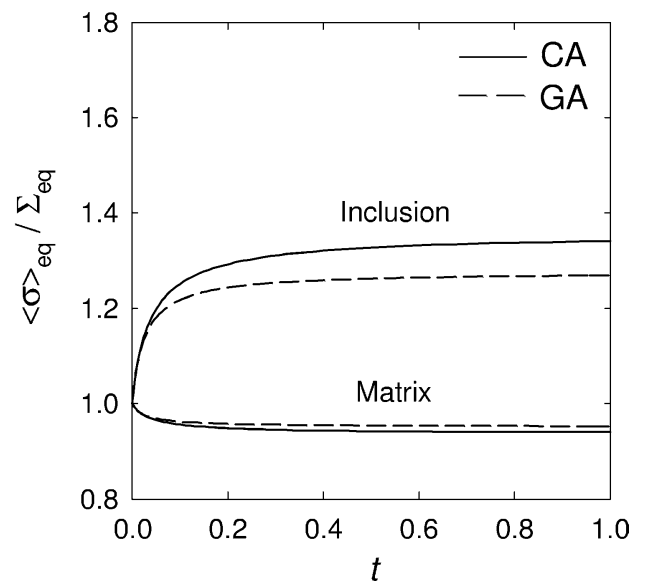

(a)

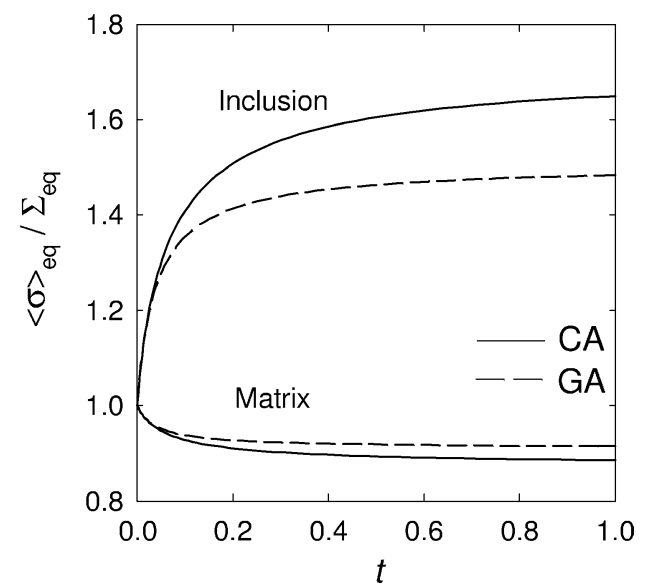

(b)

Fig. 4. Normalised equivalent average stresses per phase estimated by the classical and generalised affine procedures for (a) aligned-fiber and (b) spherical inclusions. Same material and loading condition as Fig. 3.

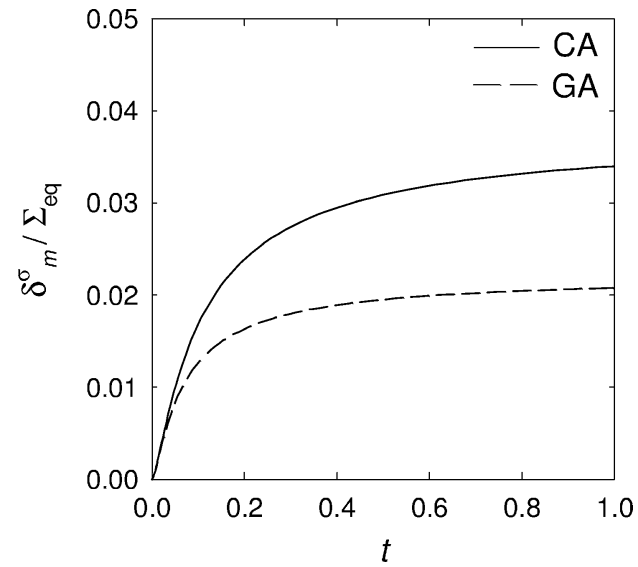

(a)

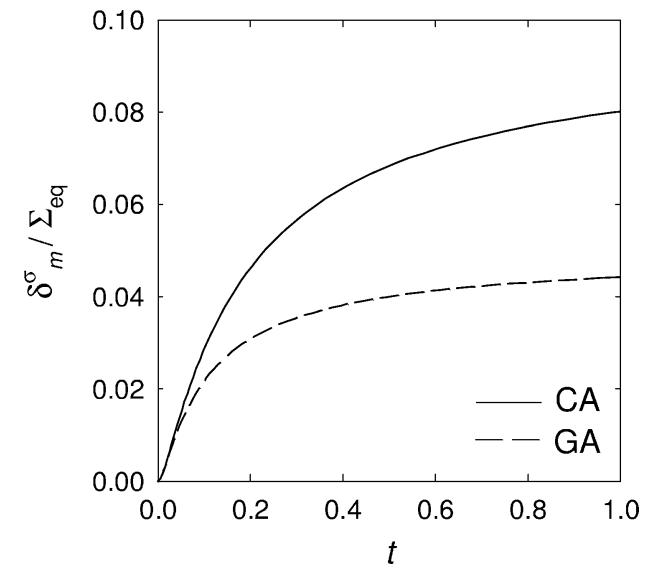

(b)

Fig. 5. Normalised stress fluctuation within the matrix estimated by the classical and generalised affine procedures for (a) aligned-fiber and (b) spherical inclusions. Same material and loading condition as Fig. 3.

homogenisation procedure. To characterise the evolution of the intraphase deviatoric stress $\mathbf{s}$ and isochoric strain $\mathbf{e}$ fluctuations within the viscoelastic matrix, it is convenient to introduce the following quantities,

$$
\delta_{m}^{\sigma}=\langle\mathbf{s}: \mathbf{s}\rangle_{2}-\langle\mathbf{s}\rangle_{2}:\langle\mathbf{s}\rangle_{2}, \quad \delta_{m}^{\varepsilon}=\langle\mathbf{e}: \mathbf{e}\rangle_{2}-\langle\mathbf{e}\rangle_{2}:\langle\mathbf{e}\rangle_{2},
$$

which, although not variances, are positive and equal to zero only if the fields are homogeneous within the matrix. They are respectively plotted in Figs. 5 and 6. Note that the corresponding quantities for the inclusions are equal to zero since the fields predicted by the Mori-Tanaka scheme are homogeneous in the inclusions. For a given procedure, aligned fibers in the shear direction induce less perturbations of the stress and strain field within the matrix than spherical inclusions isotropically distributed. This reflects at the intraphase level the relative discrepancies observed between CA and GA estimates for the overall response. Although both CA and GA estimates predict the same trends at the intraphase level, it is interesting to note that GA estimates predict higher strain fluctuations and lower stress fluctuations in the matrix regardless of the considered microstructure. It can be emphasised that a similar observation has been previously reported with the MA scheme (Brenner et al., 2001) for self-consistent estimates of the steady-state (nonlinear viscous) regime. For the shear loading path considered, it is quite intuitive that the effective strain response is rather driven by the matrix. This remark is especially true for the GA estimate which takes into account the strong fluctuations (consistent with shear bands) which occur within the matrix (see Moulinec and Suquet, 2003). 


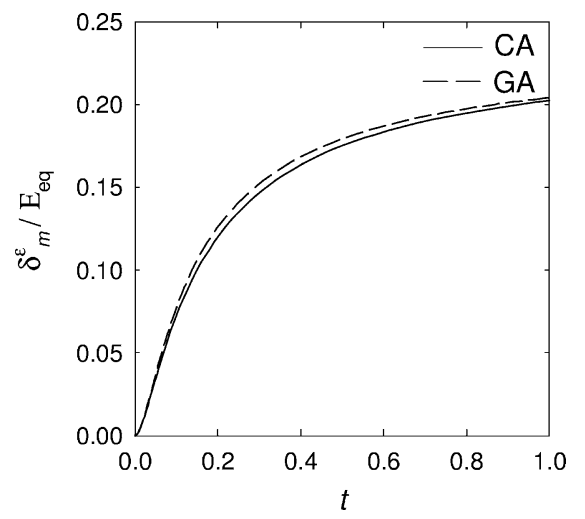

(a)

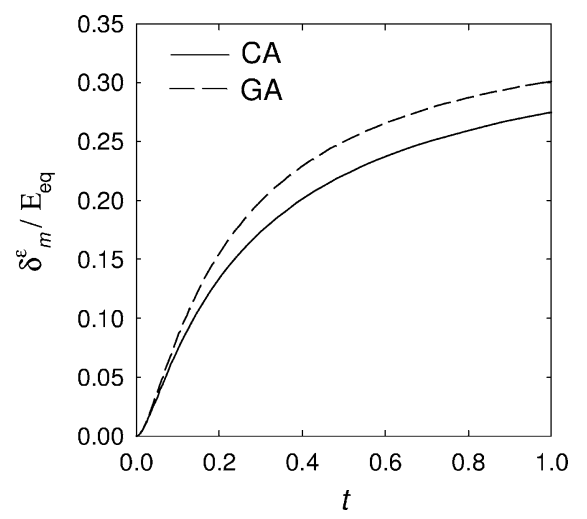

(b)

Fig. 6. Normalised strain fluctuation within the matrix estimated by the classical and generalised affine procedures for (a) aligned-fiber and (b) spherical inclusions. Same material and loading condition as Fig. 3.

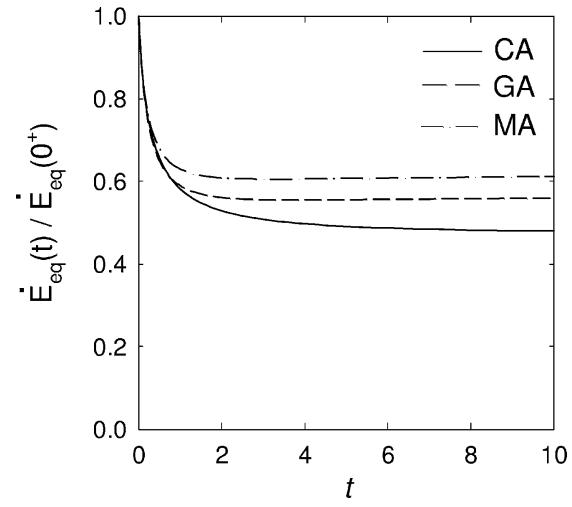

(a)

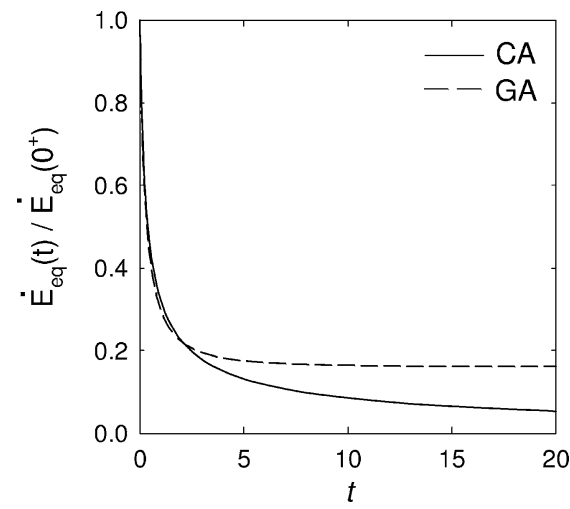

(b)

Fig. 7. Normalised overall equivalent strain rate estimated by the classical and generalised affine procedures for (a) spherical and (b) oblate ellipsoidal inclusions. The volumic fraction of inclusions is $c_{i}=0.15$ and the stress sensitivity exponent of the matrix is $n=5$. Case of uniaxial tension with $\Sigma_{33} / \sigma_{0}^{(2)}=1$.

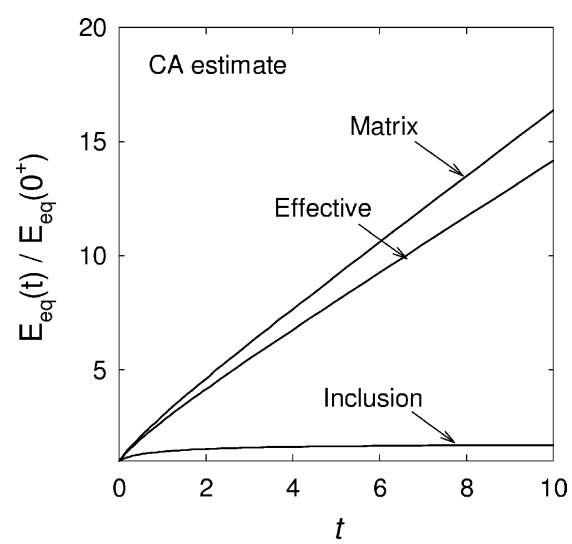

(a)

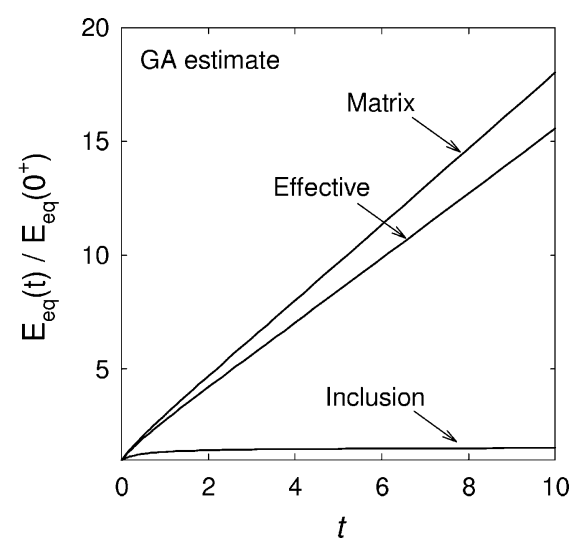

(b)

Fig. 8. Normalised equivalent average strain per phase estimated by the (a) classical and (b) generalised affine procedures for spherical inclusions. Same material and loading condition as Fig. 7. 


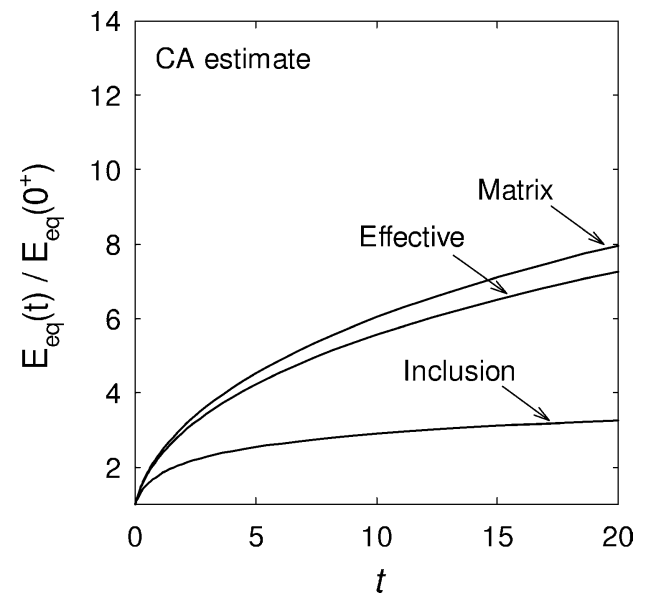

(a)

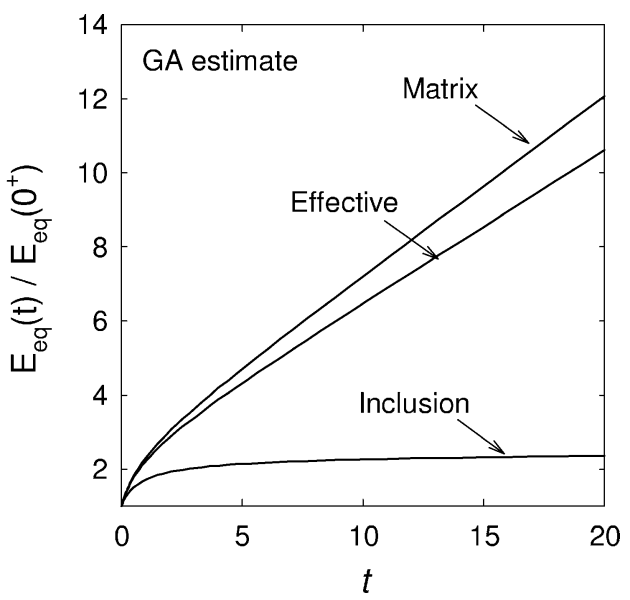

(b)

Fig. 9. Normalised equivalent average strain per phase estimated by the (a) classical and (b) generalised affine procedures for oblate ellipsoidal inclusions. Same material and loading condition as Fig. 7.

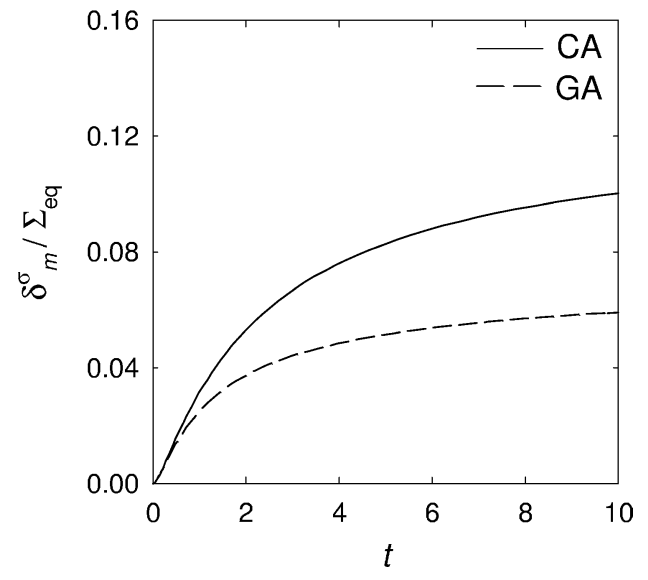

(a)

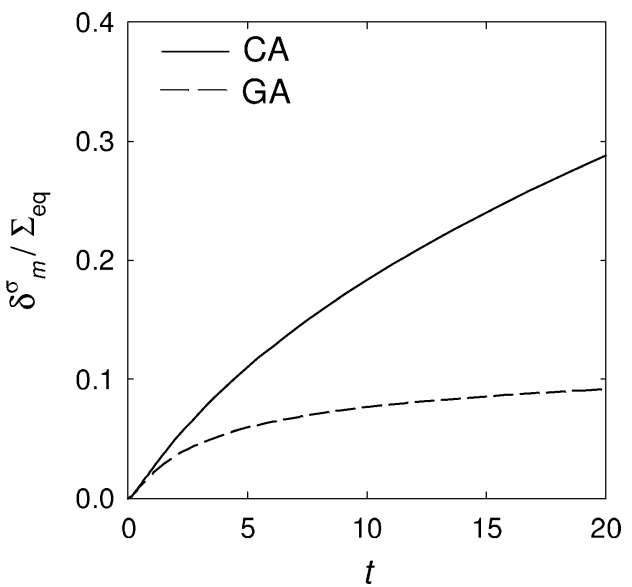

(b)

Fig. 10. Normalised stress fluctuation within the matrix estimated by the classical and generalised affine procedures for (a) spherical and (b) oblate ellipsoidal inclusions. Same material and loading condition as Fig. 7.

\subsection{Creep under uniaxial tension}

In connection with the preceding observations, the case of a uniaxial tensile loading along $x_{3}$ direction is now studied. Two kinds of inclusions are still considered, namely: spheres and oblate spheroids along $x_{3}$ axis. For the latter morphology, an aspect ratio of 5 was considered. It approximates the case of discontinuous fibers. The effective response is plotted in Fig. 7. The strong reinforcement caused by the oblate spheroidal elastic inclusions, with respect to the spherical ones, can be observed in the steady-state regime. For comparison, the MA estimate has been added for the spherical case. Interestingly, it can be noted that although leading to a different viscous regime, the predicted duration of the transient regime is very close to the one of the GA estimate. Again, it appears that the improved affine estimates present the trend of a shorter transient regime. The interphase strain fluctuations within the composite are illustrated in Figs. 8 and 9. In the case of spherical inclusions (i.e. moderate reinforcement), it can be seen that both CA and GA formulations lead to very similar responses of the matrix and the inclusions. On the contrary, for oblate inclusions, it is obvious that the CA estimate predicts a very stiff response of the matrix (and consequently the composite) compared to the GA estimates. This can be explained by the fact that the CA estimate which does not take into account the field fluctuations within the matrix will tend to predict a behaviour strongly driven by the inclusions response because of the "aligned" morphology along the tensile direction. The improvement obtained with the GA estimate is obvious in that case. The comparison of the intraphase stress fluctuations between the two morphologies (Fig. 10) 
assesses this last remark since it is obtained that oblate spheroidal inclusions lead to an increase of the stress field heterogeneity within the matrix.

\section{Concluding remarks}

In this work, we have proposed an original procedure to estimate the homogenised properties of nonlinear viscoelastic composites. Basically, this approach relies on a linearisation procedure which mixes the hereditary affine approach (Masson and Zaoui, 1999) and the variational second-order procedure (Ponte Castañeda, 2002a). Consequently, this scheme allows to derive estimates for hereditary behaviours incorporating field fluctuations. It has been compared with the classical affine approach in the context of matrix-inclusion microstructures, with homogeneous elasticity, under creep conditions. Especially, the effect of the inclusions' morphology combined with the loading path on the intraphase field fluctuations has been emphasised. Our analysis shows that accounting for the field fluctuations to estimate the homogenised behaviour leads to softer overall responses as well as a faster stress field redistribution within the material which implies a shortened viscoelastic regime.

\section{Acknowledgements}

Fruitful discussions with P. Ponte Castañeda during this work are gratefully acknowledged.

\section{References}

Bobeth, M., Diener, G., 1986. Field fluctuations in multicomponent mixtures. J. Mech. Phys. Solids 34, 1-17.

Brenner, R., Castelnau, O., Gilormini, P., 2001. A modified affine theory for the overall properties of nonlinear composites. C. R. Acad. Sci. Paris, Ser. IIb 329, 649-654.

Brenner, R., Masson, R., Castelnau, O., Zaoui, A., 2002. A "quasi-elastic" affine formulation for the homogenized behaviour of nonlinear viscoelastic polycrystals and composites. Eur. J. Mech. A Solids 21, 943-960.

Buryachenko, V.A., 1993. Effective strength properties of elastic physically nonlinear composites. In: Mecamat 93, International Seminar on Micromechanics of Materials. Eyrolles, Paris, pp. 567-578.

Buryachenko, V.A., 2001. Multiparticle effective field and related methods in micromechanics of composite materials. Appl. Mech. Rev. 54, $1-47$.

Eshelby, J.D., 1957. The determination of the elastic field of an ellipsoidal inclusion, and related problems. Proc. Roy. Soc. London Ser. A 241, 376-396.

Gilormini, P., 1995. Insuffisance de l'extension classique du modèle autocohérent au comportement non linéaire. C. R. Acad. Sci. Paris, Ser. IIb 320, 115-122.

Hill, R., 1965. Continuum micro-mechanics of elastoplastic polycrystals. J. Mech. Phys. Solids 13, 89-101.

Kreher, W., 1990. Residual stresses and stored elastic energy of composites and polycrystals. J. Mech. Phys. Solids 38, $115-128$.

Idiart, M., Ponte Castañeda, P., 2003. Field fluctuations and macroscopic properties for nonlinear composites. Int. J. Solids Struct. 40, 70157033.

Laws, N., McLaughlin, R., 1978. Self-consistent estimates for the viscoelastic creep compliances of composite materials. Proc. Roy. Soc. London Ser. A 359, 251-273.

Mandel, J., 1966. Mécanique des milieux continus. Gauthier-Villars, Paris.

Masson, R., Zaoui, A., 1999. Self-consistent estimates for the rate-dependent elastoplastic behaviour of polycrystalline materials. J. Mech. Phys. Solids 47, 3543-3568.

Masson, R., Bornert, M., Suquet, P., Zaoui, A., 2000. An affine formulation for the prediction of the effective properties of nonlinear composites and polycrystals. J. Mech. Phys. Solids 48, 1203-1227.

Mori, T., Tanaka, K., 1973. Average stress in matrix and average elastic energy of materials with misfitting inclusions. Acta Metall. Mater. 21, 597-629.

Moulinec, H., Suquet, P., 2003. Intraphase strain heterogeneity in nonlinear composites: a computational approach. Eur. J. Mech. A Solids 22, 751-770.

Ponte Castañeda, P., 1996. Exact second-order estimates for the effective mechanical properties of nonlinear composite materials. J. Mech. Phys. Solids 44, 827-862.

Ponte Castañeda, P., 2002a. Second-order homogenization estimates for nonlinear composites incorporating field fluctuations. I - Theory. J. Mech. Phys. Solids 50, 737-757.

Ponte Castañeda, P., 2002b. Second-order homogenization estimates for nonlinear composites incorporating field fluctuations. II - Applications. J. Mech. Phys. Solids 50, 759-782.

Ponte Castañeda, P., Suquet, P., 1998. Nonlinear composites. Adv. Appl. Mech. 34, 171-302.

Pouya, A., Zaoui, A., 1999. Linéarisation et homogénéisation en viscoélasticité. C. R. Acad. Sci. Paris, Ser. IIb 327, 365-370. 
Rougier, Y., Stolz, C., Zaoui, A., 1994. Self-consistent modelling of elastic-viscoplastic polycrystals. C. R. Acad. Sci. Paris, Ser. II 318, 145151.

Schapery, R.A., 1962. Approximate methods of transform inversion for viscoelastic stress analysis. In: Proc. U.S. Nat. Congr. Appl. Mech. ASME 4th, vol. 2, pp. 1075-1085.

Suquet, P., 1995. Overall properties of nonlinear composites: a modified secant moduli theory and its link with Ponte Castañeda's nonlinear variational procedure. C. R. Acad. Sci. Paris, Ser. IIb 320, 563-571. 